\title{
Intermediate-term outcome of placement of Baerveldt glaucoma implant for refractory glaucoma in a Malaysian population
}

\author{
Clarissa Ern Hui Fang, Seng Kheong Fang \\ Department of Ophthalmology, International Specialist Eye Centre, Kuala \\ Lumpur, Malaysia
}

\begin{abstract}
Objective: To report baseline characteristics and surgical outcomes of placement of Baerveldt glaucoma implant (BGI) in Asian eyes with considerably elevated intraocular pressure (IOP) despite maximal medical therapy.

Design: Retrospective case series of surgical cases from a single surgeon. Retrospective review of medical records of last clinic visits.

Participants: One hundred and ninety-seven eyes of patients underwent placement of 350- $\mathrm{mm}^{2}$ Baerveldt implant.

Methods: The medical records of consecutive patients who underwent placement of a Baerveldt 350- $\mathrm{mm}^{2}$ glaucoma drainage device (GDD) at the International Specialist Eye Centre from 2007 to 2014 were reviewed. Patients with a minimum 1-year follow-up were included. Baseline characteristics, pre-operative and post-operative IOP, number of glaucoma medications, visual acuity (VA) and complications were recorded. The pre-operative IOP is compared with the IOP at 1, 2, 3 and 5 years.

Measures: The IOP, VA, supplemental medical therapy, complications and success and failures were recorded.

Results: One hundred and ninety-seven patients were followed up at 1-year post-operation, 157 patients at 2 years, 120 at 3 years and 37 at 5 years. The mean baseline IOP of $29.2 \pm 10.6 \mathrm{mmHg}$ was significantly reduced at all time points postoperatively. Mean number of glaucoma medications was significantly lower at last follow-up than pre-operatively (1.8 vs. 2.7).

Conclusions: Placement of GDDs effectively reduces IOP without much long-term complication and may be useful in glaucomatous eyes with considerably elevated pre-operative IOP not well controlled with maximal medical therapy in the Asian population.
\end{abstract}

Keywords: Baerveldt implant, glaucoma, Malaysia, surgical outcomes

Correspondence: Clarissa Ern Hui Fang, Level 8, Centrepoint South, The Boulevard, Mid Valley City, 59200 Kuala Lumpur, Federal Territory of Kuala Lumpur, Malaysia. E-mail: fangclarissa@gmail.com 


\section{Introduction}

Glaucoma drainage implants are commonly used in the surgical management of glaucoma. Traditional indications are those with refractory or complicated glaucoma such as neovascular glaucoma, aphakic, pseudophakic, post-silicone oil glaucoma, previous multiple surgeries and previous failed trabeculectomy. With the results of the tube versus trabeculectomy, ${ }^{1}$ it is now being used earlier and may even be indicated as a primary treatment in certain cases. An example of a well-known glaucoma drainage device (GDD) is the Baerveldt glaucoma implant (BGl), a tube inserted into the anterior chamber and connected to a plate placed at the equatorial region. Success rates of such BGls range from $60 \%$ to 93\% at follow-up intervals between 14 and 37 months. ${ }^{2}$ However, many studies are on Caucasian eyes and GDDs are equally important in Asian eyes. Asian eyes tend to have increased scarring and fibrosis, thus may have potentially higher failure rate. ${ }^{3-5}$ The aim of this study was to determine the intermediate outcome of BGI surgery in Asian eyes performed in a tertiary centre in Malaysia.

\section{Materials and methods}

\section{Patients}

A retrospective review by computerized search of all patients at International Specialist Eye Centre (ISEC) Kuala Lumpur, Malaysia was carried out to identify those who underwent GDD surgery. The medical records of patients who underwent placement of a Baerveldt 350- $\mathrm{mm}^{2}$ GDD from 2007 to 2014 were reviewed. Patients with a minimum 1-year follow-up were included. Baseline characteristics, pre-operative and post-operative intraocular pressure (IOP), number of glaucoma medications, visual acuity (VA) and complications were recorded. The pre-operative IOP was compared with the IOP at 1, 2, 3 and 5 years.

\section{Procedure}

The $350-\mathrm{mm}^{2} \mathrm{BGl}$ model was used in all surgeries. As the procedure was performed by a single surgeon, the procedure and the pre-operative and post-operative management were similar in each case. Most procedures, $148(75.1 \%)$, were done under local anaesthesia, which was given as peribulbar injections with or without sedation, and the rest, 49(24.9\%), were done under general anaesthesia. Of those under local anaesthesia, all were given peribulbar injection. In some, when the peribulbar anaesthesia was not adequate, they were supplemented with sub-Tenon's injection. The preferred quadrant for surgery was supero-temporal, then supero-nasal (if there was no Baerveldt implant supero-temporally), then infero-nasal and lastly infero-temporal (to avoid vortex veins and inferior oblique muscles). Anterior chamber paracentesis 
was performed at the beginning of the surgery and slow aqueous release was performed (care especially with patients with neovascular glaucoma, and to prevent bleeding, viscoelastics may be instilled at this stage into the anterior chamber) Fornix-based conjunctival peritomy was performed, the Tenon's capsule separated and the appropriate muscle isolated and slung with a muscle hook. Mitomycin C (MMC) $0.02 \%$ soaked with Weck-Cel sponge was routinely placed under the sclera and Tenon's capsule for 3 minutes and washed away with copious amount of balanced salt solution (BSS). Haemostasis was secured with diathermy.

The $\mathrm{BGI}$ was primed to make sure the tube is patent and the plate placed under the appropriate muscle on each side and anchored with 8-0 nylon through each anchoring hole. The tube was ligated completely near its junction with the plate with 7-0 Vicryl using two throws. Care was ensured that there was no flow through this ligature. Then, at least 12 and up to 20 Sherwood venting slits were created through the tube distal to the ligature with the needle of the 7-0 Vicryl suture. The tube was then shortened and the length adjusted to be inserted about 2.5 to $3 \mathrm{~mm}$ into the anterior chamber. A tract was made through the sclera about $2 \mathrm{~mm}$ away from the limbus and entered into the anterior chamber through the angle. Care was taken that this tract was not too close to the iris and not too close to the cornea. A small amount of viscoelastics was instilled through the tract to make sure there is adequate space for the tube to be inserted. The tube was then inserted into the anterior chamber through this tract using a tube introducing forceps and the length was checked to be adequate. The tube was then anchored to the sclera with a mattress non-absorbable 10-0 nylon suture. The exposed area of the tube was then covered with donor cornea sclera (earlier preserved in glycerine, but subsequently soaked in BSS and then gentamicin eye drops) sutured with 10-0 nylon. The overlying conjunctiva was then closed with continuous absorbable 8-0 Vicryl stitch making sure the closure was watertight. Intracameral and topical antibiotic (moxifloxacin) was instilled and also topical Tobradex (dexamethasone-tobramycin combination) eye ointment (Alcon). The operated eye was padded and protected with a shield.

There were a few cases that were combined with cataract surgery with intraocular lens implant. In these cases, first the plate was inserted and then temporal clear cornea phacoemulsification cataract surgery was done with implantation of intraocular lens. Usually a single 10-0 nylon was applied to the clear cornea wound. Then finally the tube was inserted.

Patients were examined on the first post-operative day and 1 to 2 weekly (for 6 weeks) and then 1 to 3 monthly as clinically indicated. The IOP was measured with a Goldmann applanation tonometer at each visit, and glaucoma medication was added as required to supplement IOP reduction if the target IOP was 
not achieved. A few cases had laser suture lysis on average of 4 to 5 weeks post-operatively if medications were not able to control the IOP.

\section{Outcome criteria}

The surgical outcome (at last visit) was assessed in terms of IOP, VA, and the incidence of complications. The IOP at last visit was recorded. A complete success was defined as IOP of between 6 and $21 \mathrm{mmHg}$ without medication, qualified success as IOP between 6 and $21 \mathrm{mmHg}$ with one or more medications and failure as IOP more than $21 \mathrm{mmHg}$ or less than $6 \mathrm{mmHg}$ and those who lost light perception.

\section{Results}

A total of 240 patients had the BGl surgery from 2007 to 2014. Patients with less than 12-months follow-up were excluded from this study. A total of 197 patients had 1-year follow-up post-operation, 157 patients at 2 years, 120 patients at 3 years and 37 patients at 5 years.

Table 1. Demographic characteristics of patients undergoing BGI surgery

\begin{tabular}{|l|l|}
\hline Patients & Total, $\mathbf{n}=197$ \\
\hline Age & $53.6 \pm 17.8$ years (range: 6-86) \\
\hline$<20$ years & $9(4.6 \%)$ \\
\hline$>50$ years & $132(67 \%)$ \\
\hline Sex & \\
\hline Male & $141(71.6 \%)$ \\
\hline Female & $56(28.4 \%)$ \\
\hline Ethnicity & \\
\hline Chinese & $142(72.1 \%)$ \\
\hline Malay & $38(19.3 \%)$ \\
\hline Indian & $15(7.6 \%)$ \\
\hline Other & $2(1.0 \%)$ \\
\hline Operated eye & \\
\hline Right & $105(53.3 \%)$ \\
\hline Left & $92(46.7 \%)$ \\
\hline
\end{tabular}


All the patients were of Asian origin. The majority of the patients were from Malaysia (94.4\%) and the rest were from Indonesia, Singapore and Saudi Arabia.

The patients consisted of 142 Chinese (72.1\%), 38 Malays (19.3\%), 15 Indians $(7.6 \%)$ and 2 other (1.0\%). The demographic features of the patients are summarized in Table 1.

The most common diagnosis for surgery was neovascular glaucoma; there were 51 eyes (25.9\%). Neovascular glaucoma is prevalent because diabetes is very common in Malaysia and ISEC is tertiary referral centre. Many of the eyes with proliferative diabetic retinopathy have had previous intra-vitreal anti-VEGF or laser photocoagulation with signs of regression of the neovascularization before BGI surgery. However, some patients with poor media that precluded laser therapy or IOP too high with maximal medical therapy either underwent pre-operative intra-vitreal anti-VEGF or concomitant intra-operative intra-vitreal anti-VEGF and $\mathrm{BGI}$. The second most common diagnosis was pseudophakia or aphakia in 48 eyes

Table 2. Primary pre-operative diagnosis of patients undergoing BGI surgery

\begin{tabular}{|l|l|}
\hline Type of glaucoma & \\
\hline Neovascular glaucoma & 51 \\
\hline Pseudophakia/aphakia & 48 \\
\hline Primary open-angle glaucoma with previous failed trabeculectomy & 37 \\
\hline Uveitic glaucoma & 19 \\
\hline Post-keratoplasty & 10 \\
\hline Juvenile & 7 \\
\hline Post-traumatic & 5 \\
\hline Steroid-induced & 5 \\
\hline Congenital & 4 \\
\hline Post-retinal detachment & 3 \\
\hline Iridocorneal endothelial syndrome & 3 \\
\hline Primary angle closure & 2 \\
\hline Sturge-Weber syndrome & 1 \\
\hline Silicone oil glaucoma & 1 \\
\hline Pseudoexfoliation & 1 \\
\hline
\end{tabular}


(24.4\%), mostly after complicated cataract surgery. BGI was the primary procedure for those refractory cases such as neovascular, uveitic, post-retinal detachment (RD)/silicone oil, post-corneal graft, some pseudophakic/aphakic and iridocorneal endothelial syndrome. The primary pre-operative diagnoses are listed in Table 2.

Table 3 summarizes the mean IOP before and after BGI surgery at 1, 2, 3 and 5 years. IOP was reduced from a mean pre-operative IOP of $29.2 \pm 10.6$ to $14.7 \pm 4.4$ after 1 year, a reduction of approximately $49.7 \%$. After 2 years, the mean IOP was $14.4 \pm 4.4$ (50.7\% reduction), after 3 years it was $15.0 \pm 3.9$ and after 5 years it was $15.5 \pm 4.3$. Student's paired t-tests were carried out which showed that there is a difference between pre-operative IOP and post-operative IOP. The $p$-value is 0.0001 in all comparisons, showing that the difference is significant. This is shown in Table 4.

Overall outcomes for BGI in terms of IOP: 47 eyes (23.9\%) were classified as complete successes, 144 eyes (73.1\%) were qualified successes as they continued medication to control the IOP and $6(3.0 \%)$ were failures. Of the failures, two eyes $(1 \%)$ had no perception to light; four eyes (1.5\%) had IOP levels more than $21 \mathrm{mmHg}$, which had further glaucoma surgeries; three eyes had second glaucoma implants and one eye had transscleral cyclophotocoagulation. Table 5 summarises the overall outcomes for BGl.

Table 3. IOP before and after BGI surgery

\begin{tabular}{|l|l|l|}
\hline IOP & Mean(SD) & N \\
\hline Pre-operation & $29.2(10.6)$ & 197 \\
\hline 1 year & $14.7(4.4)$ & 197 \\
\hline 2 years & $14.4(4.4)$ & 158 \\
\hline 3 years & $15.0(3.9)$ & 120 \\
\hline 5 years & $15.5(4.3)$ & 37 \\
\hline
\end{tabular}

Table 4. IOP comparisons before and after BGI surgery

\begin{tabular}{|l|l|l|l|}
\hline $\begin{array}{l}\text { Pre-operation } \\
\text { IOP compared to }\end{array}$ & $\begin{array}{l}\text { Paired differences } \\
\text { mean }\end{array}$ & P-value & $\begin{array}{l}\mathbf{9 5 \%} \text { Confidence } \\
\text { interval }\end{array}$ \\
\hline IOP at 1 year & 14.4 & 0.0001 & $12.8603-16.0128$ \\
\hline IOP at 2 year & 14.3 & 0.0001 & $12.5779-16.0677$ \\
\hline IOP at 3 year & 14.6 & 0.0001 & $12.6028-16.6138$ \\
\hline IOP at 5 year & 14.6 & 0.0001 & $11.3983-17.8450$ \\
\hline
\end{tabular}


Table 5. Overall outcome for BGI surgery

\begin{tabular}{|l|c|}
\hline Outcome at last visit & \\
\hline Complete success (IOP 6-21 mmHg; no medication: not NPL) & $47(23.9 \%)$ \\
\hline Qualified success (IOP 6-21 mmHg; with medication: not NPL) & $144(73.1 \%)$ \\
\hline $\begin{array}{l}\text { Failure } \\
\text { IOP }>21 \mathrm{mmHg} \text {, IOP }<6 \mathrm{mmHg}\end{array}$ & $6(3.0 \%)$ \\
NPL at last visit & \\
\hline
\end{tabular}

NPL, no perception to light

Table 6. Post-operative VA outcomes of BGI surgery

\begin{tabular}{|l|l|l|l|l|}
\hline VA & $\mathbf{1}$ year & $\mathbf{2}$ years & $\mathbf{3}$ years & $\mathbf{5}$ years \\
\hline Improved $^{\mathrm{a}}$ & $34.5 \%$ & $34.3 \%$ & $27.0 \%$ & $26.3 \%$ \\
\hline Unchanged & $34.5 \%$ & $38.2 \%$ & $27.0 \%$ & $31.6 \%$ \\
\hline Worsened $^{\mathrm{a}}$ & $31.0 \%$ & $27.7 \%$ & $45.9 \%$ & $42.1 \%$ \\
\hline
\end{tabular}

aBy one or more lines of Snellen acuity

Table 6 summarises the change in VA. The VA at clinic visit was compared to pre-operative VA. There was an improvement of VA in most patients after 1 year. However, the percentage decreases as the number of years post-operation increases.

The majority of the patients are on fewer medications than they were preoperatively. This is consistent for each year. The mean number of pre-operative medication was 2.7 and the number of medication post-operation at 1 year, 2 years, 3 years and 5 years were 1.8, 1.7, 1.8 and 1.8 , respectively; this is shown in Table 7.

Table 7. Number of medications before and after BGI surgery

\begin{tabular}{|l|l|}
\hline Number of medications & Mean \pm SD (range) \\
\hline Pre-operative & $2.7 \pm 1.2(0-5)$ \\
\hline Post-operative (1 year) & $1.8 \pm 1.4(0-4)$ \\
\hline Post-operative (2 years) & $1.7 \pm 1.4(0-4)$ \\
\hline Post-operative (3 years) & $1.8 \pm 1.5(0-4)$ \\
\hline Post-operative (5 years) & $1.8 \pm 1.5(0-4)$ \\
\hline
\end{tabular}




\section{Complications}

The most common intra-operative complication in the surgery was bleeding (eight patients), the most common early post-operative complication was hyphema (three patients) and the most common late complication was bullous keratopathy. The post-operative complications are listed in Table 8, grouped into early and late complications.

Table 8. Early and late post-operative complications from BGI surgery

\begin{tabular}{|l|l|}
\hline Early complications & \\
\hline Hyphema & 3 \\
\hline Choroidal detachment & 2 \\
\hline Endophthalmitis & 1 \\
\hline Tube blockage & 1 \\
\hline Late complications & \\
\hline Bullous keratopathy & 3 \\
\hline Tube erosion & 1 \\
\hline Tube blockage & 1 \\
\hline Cystoid macular oedema & 1 \\
\hline
\end{tabular}

\section{Discussion}

This study has shown that the intermediate- to long-term results of the BGl in Asian population is satisfactory in the majority of the cases. There was a significant decrease in IOP at each follow-up visit at 1, 2, 3 and 5 years. These results are promising because of failure of medical management and poorer success of trabeculectomy augmented with antimetabolite.

There was improvement of VA in many patients after 1 year. However, the percentage decreases as the number of years post-operation increases. This suggests that the $\mathrm{BGl}$ can improve VA, but this improvement isn't in the long term. However, many of the patients would have lost visual potential and have continued deterioration because of the severity of their glaucoma at presentation. There were numerous other causes of further deterioration of vision other than from the glaucoma after 1 year, but these were not analysed. Most commonly patients developed cataracts, or cystoid macula oedema or have progression of their diabetic retinopathy 
Generally, the incidence of post-operative complications were low. This exceeded expectations compared to previous reports. In total, 184 eyes (93.4\%) did not have any post-operative complications with the BGl surgery. The most common intra-operative complications in the surgery was bleeding, in 8 cases out of 197 eyes (4.1\%), most of which are from patients with neovascular glaucoma. The most common early complication was hyphema (three eyes), which resolved spontaneously after corticosteroid eye drops in 1 to 2 weeks. These cases were in those with pre-existing neovascularization and post-traumatic cases. Hyphema was prevented by slow decompression of eye during surgery, tamponade with viscoelastics during and at the end of surgery.

There was a low rate of choroidal detachment (two eyes) and suprachoroidal haemorrhage (none). In the two cases of choroidal detachment, they were due to post-operative hypotony and this has a higher incidence in previously vitrectomized eyes. Precautionary measures were taken to prevent early post-operative hypotony by ligation of tube, and instillation of viscoelastic in anterior chamber intra-operatively. One eye was treated conservatively with cycloplegic drops and resolved spontaneously, and in the other one, the anterior chamber was reformed with viscoelastic injection. None required surgical drainage.

There was one case of endophthalmitis where this patient had malignant glaucoma post-operatively, had core vitrectomy, then developed endophthalmitis, but resolved with oral and intra-vitreal antibiotics. Strict precautions were taken in all cases to prevent this complication such as good diabetic control, strictly aseptic surgical technique and intracameral and post-operative topical and oral antibiotics were routinely given.

Another complication was tube blockage; there were one case each of tube blockage in the early and late stage. Common causes are blockage due to blood, vitreous, iris or silicone oil. In both cases, the blockage was due to vitreous and had YAG laser done to clear blockage. Unlike previous series, there were no complications of wound dehiscence or flat anterior chamber; these were avoided with meticulous surgical technique.

A common late complication in this study was bullous keratopathy (three eyes). These patients had previous multiple intraocular surgeries. Precautions to prevent these complications were proper surgical technique, protection of corneal endothelium with viscoelastics and proper placement of tube away from cornea. Those with this complication were treated with steroid drops and hypertonic saline and referred to corneal surgeon for further management such as corneal transplant. One eye had cystoid macula oedema and resolved after treatment with topical non-steroidal anti-inflammatory drugs eye drops (nepafenac). Lastly, tube erosion (one eye) was managed successfully with surgical revision of wound with sclera and conjunctival graft. 
One of the reasons for the lower rate of complications and longer-term success could be attributed to the surgical technique used in this study, which is slightly different from others. Mitomcyin $C$ was routinely used in most cases, $0.2 \mathrm{mg} / \mathrm{ml}$ soaked in Weck-Cell sponge and placed between Tenon's capsule and sclera for 3 minutes and washed away by copious amount of balance salt solution. This would prevent future fibrosis and encapsulation over the plate. The number of Sherwood slits was more than the recommended, but it was done based on the surgeon's experience and the amount of viscoelastic gel left in the anterior chamber, thus preventing major immediate post-operative complications.

Comparing this study's success rate with other studies, reduction in IOP at 2 years was $50.7 \%$, which is comparable to other studies at 2 years. This is similar to BGI in a study by Krishna et al. (56\%), Ahmed implants (51\%), double-plate Molteno implants (46-53\%). This is higher than that reported with single-plate Molteno implants (25\%) but lower than that reported with Krupin devices (64\%)., ${ }^{2,6}$

$\mathrm{BGl}$ has a bigger but flatter plate compared to other devices but is non-valved. Because of its bigger surface area, it potentially will have higher long-term rate of success, although there is not a lot in the literature about long-term outcomes. It is more difficult to implant compared to the other GDDs like Ahmed, because it is a bigger plate and is non-valved, it needed measures to prevent initial hypotony, such as tube ligation and stenting and Sherwood slits., Initial studies shows that the success increases with the size of the plate, but up to about $500 \mathrm{~mm}^{2}$. There are three sizes available, $250 \mathrm{~mm}^{2}$ for young children, $500 \mathrm{~mm}^{2}$ plate is too big and difficult to implant. The choice of $350 \mathrm{~mm}^{2}$ was because of surgeon preference.

The Ahmed and Baerveldt $(A B C)$ comparison study showed that with the $B G l$, success rates were higher and patients achieved both a statistically significant lower IOP $(p=0.015)$ and a trend toward statistical significance $(p=0.28)$ for reduced need for glaucoma medication compared to the Ahmed implant. ${ }^{8}$ Ahmed vs Baerveldt (AVB) study showed that both devices were effective in reducing IOP and glaucoma medications. The Baerveldt group had a lower failure rate and required fewer medications than the Ahmed group after 3 years, but it experienced more hypotony-related vision-threatening complications. ${ }^{9}$

The results of the Tube versus Trabeculectomy study showed that patients with medically uncontrolled glaucoma who had previous cataract extraction with intraocular lens implantation and/or failed filtering surgery who were treated with tube shunt surgery were more likely to maintain IOP control and avoid persistent hypotony, loss of light perception vision or re-operation for glaucoma in comparison to those who underwent trabeculectomy with MMC. ${ }^{1,10}$

This study shows encouraging results of long-term effects of BGI on the IOP. The mean IOP at 3 and 5 years were 15.0 and 15.5, a reduction of $48.6 \%$ and $46.9 \%$ respectively, compared to pre-operation IOP. This shows that the IOP remains low 
even in the long term. Overall success is excellent with only $3 \%$ failures. Of the failures, two eyes (1\%) had no perception to light and four eyes (2\%) had IOP levels more than $21 \mathrm{mmHg}$. Of those eyes, three eyes had subsequent second glaucoma implant surgeries with $\mathrm{BGl}$. One eye $(0.5 \%)$ subsequently had transscleral cyclophotocoagulation. None required bleb revision or needling.

With regard to the long-term success of BGl, some may last till the patient's lifetime, although over time, encapsulation of fibrous tissue over the plate causes increased resistance to flow. This study shows encouraging longer-term results in terms of IOP.

MMC augmented trabeculectomy is reported to be less successful than GDD. Studies regarding cyclophotocoagulation (TCP) show promise but potentially has more complications such as phthisis and is reserved for end-stage cases such as those with painful blind eyes and poor prognosis for vision. In this study, the decision to implant BGI was made for refractory glaucoma, where trabeculectomy will likely fail but patient still has visual potential. Those with no visual potential, transscleral cyclophotocoagulation (cyclodestructive procedure) was advised.

There is difficulty in comparison of intermediate-term and long-term follow-up studies because of lack of uniform success criteria, exclusion of high-risk patients and inclusion of patients with short-term follow-up. Our study is unique in that it attempts to address these concerns by including patients up to 5 years of follow-up, and including all types of pre-operative diagnoses to ascertain true intermediate- to long-term follow-up results. Similar to other studies, there is difficulty achieving long-term follow-up because many patients returned to their referring ophthalmologist for long-term follow-up. The limitations of this non-randomized retrospective with variable follow-up is that the data on optic nerve and visual field progression was difficult to obtain It was also unique that it was a large series of Asian patients which is valuable. The good intermediateto long-term outcomes of BGls in Asian eyes are encouraging for those with complicated and refractory glaucoma. The BGl appears to be an effective means of lowering IOP in patients with complicated glaucoma in Asian eyes.

The collection of data for this study was in 2015, and it will be good to know the 10-years outcome of some of those patients who had their BGI in 2007. These results will be published in the future.

\section{References}

1. Gedde S, Schiffman J, Feuer W, Herndon L, Brandt J, Budenz D. Treatment outcomes in the tube versus trabeculectomy (TVT) study after five years of follow-up. Am J Ophthalmol. 2012;153(5):789-803.e2. doi:10.1016/j.ajo.2011.10.026.

2. Hodkin M, Goldblatt W, Burgoyne C, Ball S, Insler M. Early clinical experience with the Baerveldt implant in complicated glaucomas. Am J Ophthalmol. 1995;120(1):32-40. doi:10.1016/ s0002-9394(14)73756-0. 
3. Aung T, Seah S. Glaucoma drainage implants in Asian eyes. Ophthalmology. 1998;105(11):21172122. doi:10.1016/s0161-6420(98)91136-8.

4. Husain R, Clarke J, Seah S, Khaw P. A review of trabeculectomy in East Asian people-the influence of race. Eye. 2004;19(3):243-252. doi:10.1038/sj.eye.6701477.

5. Tsai A, Boey $\mathrm{P}$, Htoon $\mathrm{H}$, Wong T. Bleb needling outcomes for failed trabeculectomy blebs in Asian eyes: a 2 year follow up. Acta Ophthalmol. 2014;92:0-0. doi:10.1111/j.1755-3768.2014.t047.x.

6. Krishna R, Godfrey D, Budenz D, et al. Intermediate-term outcomes of 350-mm2 Baerveldt glaucoma implants. Ophthalmology. 2001;108(3):621-626. doi:10.1016/s0161-6420(00)00537-6.

7. Seah S, Gazzard G, Aung T. Intermediate-term outcome of Baerveldt glaucoma implants in Asian eyes. Ophthalmology. 2003;110(5):888-894. doi:10.1016/s0161-6420(03)00088-5.

8. Rockwood E. The Ahmed Baerveldt Comparison (ABC) study: long-term results, successes, failures, and complications. Am J Ophthalmol. 2016;163:xii-xiv. doi:10.1016/j.ajo.2015.12.031.

9. Christakis P, Ahmed I. The Ahmed Versus Baerveldt (AVB) study. US Ophthal Rev. 2017;10(01):21. doi:10.17925/usor.2017.10.01.21.

10. Gedde S. Results from the Tube versus Trabeculectomy. Middle East Af J Ophthalmol. 2009;16(3):107. doi:10.4103/0974-9233.56219. 